\title{
Optimized Waveform Relaxation Solution of RLCG Transmission Line Type Circuits
}

\author{
Mohammad D. Al-Khaleel \\ Yarmouk University \\ Jordan \\ Email: khaleel@yu.edu.jo
}

\author{
Martin J. Gander \\ University of Geneva \\ Switzerland \\ Email:Martin.Gander@unige.ch
}

\author{
Albert E. Ruehli \\ Missouri U. of Science and Techn. \\ Rolla, MO, USA \\ Email:albert.ruehli@gmail.com
}

\begin{abstract}
Today, parallel processing is necessary for the solution of large systems of ordinary differential equations (ODEs) as they are obtained from large electronic circuits or from discretizing partial differential equations (PDEs). Using a fine mesh in the discretization of these problems also leads to large compute times and large storage requirements. The waveform relaxation (WR) technique, which is ideally suited for the use of multiple processors for problems with multiple time scales has been used to solve such problems on parallel processors for such large systems of ODEs. However, applying the so-called classical WR techniques to strongly coupled systems leads to non-uniform slow convergence over a window in time for which the equations are integrated. In this paper, we present a so-called optimized WR algorithm applied to transmission line circuit problems based on the longitudinal partitioning into segments. This greatly improves the convergence for strongly coupled RLCG transmission line (TL) type circuits. The method can be applied to other similar circuits. The method is based on optimal parameters that lead to the optimal convergence of the iterations. Here, we present a practical optimized WR algorithm which is easy to use and is computationally inexpensive.
\end{abstract}

\section{INTRODUCTION}

The approach we use for decomposing large systems of ODEs into smaller decoupled subsystems is the waveform relaxation (WR) algorithm which consists of decoupling the system into smaller dynamical subcircuits. The WR method was first introduced for time-domain analysis of nonlinear dynamical systems, in particular, very large-scale integrated (VLSI) circuits in Lelarasmee et. al. [1] and [2]. The WR technique has the potential to become a very useful approach for the transient analysis for VLSI MOSFET and other types of circuits, due to the favorable numerical properties and the potential speed and accuracy improvements. Early surveys of the so-called classical algorithms with emphasis on the simulation of VLSI circuits can be found in [3] and [4]. Since then the WR algorithms have evolved to the solution of circuits and PDEs in parallel. The WR approach was applied to a multitude of problems in the circuit theory area, see for example [4]-[6], and in the partial differential equations of evolution type area, see for example [7]-[9] and references therein. In all of the WR techniques, the processors exchange appropriate waveforms between subsystems or subcircuits, and the natural coupling between blocks of components of the circuit (system) is preserved by so-called transmission conditions. However, as we will show, the convergence of the WR algorithm depends on the transmission conditions. Further, the work on PDEs shows that the classical transmission conditions are far from optimal. For challenging, highly coupled problems, more efficient transmission conditions are required which exchange additional information. For PDEs, the work by Gander et. al. in [10]-[13] and references therein has led to an important optimization process. In the circuit domain, improvement has been pursued since the original WR work using techniques such as overlap, etc., to enhance the transmission of information across the interfaces between subcircuits, e.g. [14]. However, none of them included the optimization process which was introduced originally in the PDEs work. Gander and Ruehli were the first to introduce optimized transmission conditions for the circuit domain. This was an essential step for the convergence of the WR algorithm for strongly coupled circuits such as directly coupled TL circuits. They demonstrated that this new technique can be applied effectively to diffusive circuits in [15] and to TL circuits in [16], without overlapping subcircuits. These new techniques are called optimized waveform relaxation (oWR) algorithms since they are based on an optimization process.

In recent work on TL circuits [17] and references therein, overlapping subcircuits, which relate to overlapping subdomains in domain decomposition, e.g., [12], were considered. Also, practical oWR algorithms were proposed, leading to further convergence improvements. Further, the oWR approach was applied in [18], [19] to electromagnetic and circuit problems which shows that a multitude of circuit problems can be solved. For Maxwell's equations in the frequency domain, see [20]-[24]. Also, the classical WR has been applied to new problems. New work on TL circuits [25] shows that the cWR approach leads to major improvements for multiple coupled transmission lines by transverse partitioning using cWR. In this case, the weak and limited line-to-line coupling is exploited. It is clear that these works are only the beginning of problems which can be solved using WR. So far, longitudinal and transverse partitioning have not been applied together for TLs. In [17], only single TLs were considered. However, all the TL work lays the foundation for further work in this area. In [17], the authors considered a transverse electro-magnetic (TEM) mode type TL model in which the conductance $G$ was ignored. Today, such losses are important especially for high performance circuits. For example, for VLSI designs [26], 
RLC models are incomplete if the dielectric leakage $G$ is not included. Hence, in this paper we extend the oWR technique to RLCG models. In Section 2 we introduce cWR and oWR algorithms for the RLCG model problem. We give numerical experiments in Section 3, and we conclude in Section 4.

\section{WR AlgORITHMS FOR RLCG TRANSMISSION LINES}

We analyze in this section WR algorithms applied to the RLCG TL model circuit shown in Fig. 1. Assuming the RLCG TL model circuit has $n$ sections per unit length and that the circuit is infinitely long, the modified nodal analysis (MNA) circuit equations can be simplified to the system of ODEs

$$
\dot{\boldsymbol{x}}=\left(\begin{array}{cccccccc}
\ddots & \ddots & \ddots & & & & & \\
& a & b & -a & & & & \\
& & -c & \tilde{b} & c & & & \\
& & & a & b & -a & & \\
& & & & -c & \tilde{b} & c & \\
& & & & & \ddots & \ddots & \ddots
\end{array}\right) \boldsymbol{x}+\boldsymbol{f}
$$

where the solution vector $\boldsymbol{x}=\left(\ldots, x_{-1}, x_{0}, x_{1}, \ldots\right)^{T}$ is ordered in a form such that nodal voltages alternate with currents. The odd index rows, which have $c$ and $\tilde{b}$ elements, correspond to voltage unknowns, whereas the even index rows, which have $a$ and $b$ elements, correspond to current unknowns. The constant entries of the matrix are given by $a=\frac{1}{L_{i}}$, $b=-\frac{R_{i}}{L_{i}}, \tilde{b}=-\frac{G_{i}}{C_{i}}$, and $c=-\frac{1}{C_{i}}$, where the characteristic electronic component parameters are $R_{i}=\frac{R}{n}, L_{i}=\frac{L}{n}$, $C_{i}=\frac{C}{n}$, and $G_{i}=\frac{G}{n}$, and the circuit elements per unit length, $R, L, C$, and $G$, are positive constants. The source term on the right hand side is given by the vector of functions $\boldsymbol{f}(t)=\left(\ldots, f_{-1}(t), f_{0}(t), f_{1}(t), \ldots\right)^{T}$, and we need an initial condition $\boldsymbol{x}(0)=\left(\ldots, x_{-1}^{0}, x_{0}^{0}, x_{1}^{0}, \ldots\right)^{T}$. Since the circuit is infinitely large, we need to assume that all unknowns are bounded as we move towards the ends of the circuit to have a well posed problem. We partition the circuit at an odd index row, i.e., at a row corresponding to a voltage unknown, into two subcircuits (subsystems) with overlap which ensures that both types of variables are covered. We call the unknowns in the first subsystem $\boldsymbol{u}(t)$ and in the second subsystem, we call them $\boldsymbol{w}(t)$ for $t \in[0, T]$, where $T$ is the end of the transient analysis interval. It should be noted that similar WR algorithms could be developed for other choices of partitioning and overlap. The cWR algorithm is given by

$$
\begin{aligned}
\dot{\boldsymbol{u}}^{k+1} & =\left[\begin{array}{cccc}
\ddots & \ddots & \ddots & \\
& a & b & -a \\
& & -c & \tilde{b}
\end{array}\right] \boldsymbol{u}^{k+1}+\left(\begin{array}{c}
\vdots \\
f_{-2} \\
f_{-1}
\end{array}\right)+\left(\begin{array}{c}
\vdots \\
0 \\
c w_{0}^{k}
\end{array}\right), \\
\dot{\boldsymbol{w}}^{k+1} & =\left[\begin{array}{cccc}
\tilde{b} & c & & \\
a & b & -a & \\
& \ddots & \ddots & \ddots
\end{array}\right] \boldsymbol{w}^{k+1}+\left(\begin{array}{c}
f_{-1} \\
f_{0} \\
\vdots
\end{array}\right)+\left(\begin{array}{c}
-c u_{-2}^{k} \\
0 \\
\vdots
\end{array}\right),
\end{aligned}
$$

with corresponding initial conditions $\boldsymbol{u}^{k+1}(0)$ and $\boldsymbol{w}^{k+1}(0)$. To start the WR iteration, some initial waveforms $\boldsymbol{u}^{0}(t)$ and $\boldsymbol{w}^{0}(t)$ are used. We replace now the classical transmission conditions, $u_{0}^{k+1}=w_{0}^{k}, w_{-2}^{k+1}=u_{-2}^{k}$, by the new ones

$u_{0}^{k+1}+\alpha u_{-1}^{k+1}=w_{0}^{k}+\alpha w_{-1}^{k}, w_{-1}^{k+1}+\beta w_{-2}^{k+1}=u_{-1}^{k}+\beta u_{-2}^{k}$,

which implies the classical transmission conditions at convergence if $\alpha \neq \beta$, see [16]. Using the new transmission conditions, the new oWR algorithm is given by

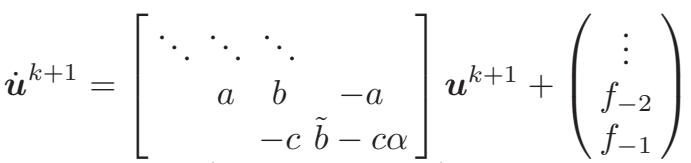

$$
\begin{aligned}
& +\left(\begin{array}{c}
\vdots \\
0 \\
c\left(w_{0}^{k}+\alpha w_{-1}^{k}\right)
\end{array}\right) \text {, } \\
& \dot{\boldsymbol{w}}^{k+1}=\left[\begin{array}{cccc}
\tilde{b}+\frac{c}{\beta} & c & & \\
a & b & -a & \\
& \ddots & \ddots & \ddots
\end{array}\right] \boldsymbol{w}^{k+1}+\left(\begin{array}{c}
f_{-1} \\
f_{0} \\
\vdots
\end{array}\right) \\
& +\left(\begin{array}{c}
-\frac{c}{\beta}\left(u_{-1}^{k}+\beta u_{-2}^{k}\right) \\
0 \\
\vdots
\end{array}\right) .
\end{aligned}
$$

Comparing the new transmission conditions with the classical ones, we can see that the new ones exchange more information by exchanging a combination of voltage and current in both directions. Further, the new ones have weighting factors $\alpha$ and $\beta$ which are part of the WR equations and can be used to optimize the performance of the new WR algorithm. By linearity of the above systems of ODEs, we consider the homogeneous problem, $\boldsymbol{f}(t)=\mathbf{0}$ and $\boldsymbol{x}(0)=\mathbf{0}$. Based on the Laplace transform with parameter $s=\sigma+i \omega \in \mathbb{C}$, and the maximum principle for analytic complex functions, we have done a mathematical analysis, which is beyond the scope of this short paper, for the convergence study. This analysis leads to the convergence factors

$$
\begin{aligned}
& \rho_{c l a}=\left\{\begin{array}{l}
\mu_{-}^{2},\left|\mu_{+}\right|>1, \\
\mu_{+}^{2},\left|\mu_{+}\right|<1,
\end{array}\right. \\
& \rho_{\text {opt }}=\left\{\begin{array}{l}
\frac{(s-\tilde{b}) \mu_{-}+\alpha c\left(\mu_{-}-1\right)}{(s-\tilde{b})+\alpha c\left(1-\mu_{-}\right)} \cdot \frac{c\left(1-\mu_{-}\right)+\beta(s-\tilde{b}) \mu_{-}}{c\left(\mu_{-}-1\right)+\beta(s-\tilde{b})},\left|\mu_{+}\right|>1, \\
\frac{(s-\tilde{b}) \mu_{+}+\alpha c\left(\mu_{+}-1\right)}{(s-\tilde{b})+\alpha c\left(1-\mu_{+}\right)} \cdot \frac{c\left(1-\mu_{+}\right)+\beta(s-\tilde{b}) \mu_{+}}{c\left(\mu_{+}-1\right)+\beta(s-\tilde{b})},\left|\mu_{+}\right|<1,
\end{array}\right.
\end{aligned}
$$

for the cWR and oWR algorithms, respectively, and $\mu_{ \pm}$are given by

$$
\mu_{ \pm}=\frac{2 a|c|+(|\tilde{b}|+s)(|b|+s) \pm \sqrt{(2 a|c|+(|\tilde{b}|+s)(|b|+s))^{2}-4 a^{2}|c|^{2}}}{2 a|c|} .
$$

For convergence, one needs the modulus of the convergence factors, $\left|\rho_{\text {cla }}\right|$ and $\left|\rho_{\text {opt }}\right|$, be less than one for $\mathcal{R}(s) \geq 0$, and for fast convergence one needs $\left|\rho_{\text {cla }}\right| \ll 1$ for the cWR algorithm, and $\left|\rho_{\text {opt }}\right| \ll 1$ for the new WR algorithm. Clearly, $\rho_{c l a}$ is a fixed function of the circuit elements and thus the cWR algorithm does not have any adjustable parameters to be chosen in order to improve the performance. Therefore, we can only analyze for the cWR algorithm if $\left|\rho_{\text {cla }}\right|<1$ is satisfied. In contrast, $\left|\rho_{\text {opt }}\right|$ includes parameters $\alpha$ and $\beta$ 


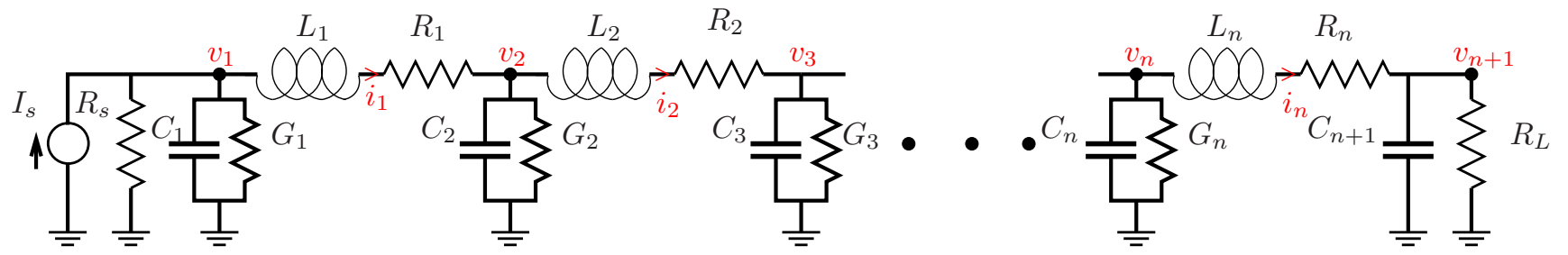

Fig. 1. Single RLCG TL model.

that can be chosen to greatly accelerate the convergence of the new WR algorithm. The optimal values for $\alpha$ and $\beta$ that make the convergence factor $\rho_{\text {opt }}$ vanish and lead to an optimal convergence in two iterations are

$$
\begin{array}{rlrl}
\alpha & =\frac{(s-\tilde{b}) \mu_{-}}{c\left(1-\mu_{-}\right)}, \beta=-\frac{c\left(1-\mu_{-}\right)}{(s-\tilde{b}) \mu_{-}}, & \left|\mu_{+}\right|>1, \\
\alpha=\frac{(s-\tilde{b}) \mu_{+}}{c\left(1-\mu_{+}\right)}, \beta=-\frac{c\left(1-\mu_{+}\right)}{(s-\tilde{b}) \mu_{+}}, & \left|\mu_{+}\right|<1 .
\end{array}
$$

As one can see, the aforementioned optimal choices are very complex and they are expensive to be used. Therefore, we look for approximations of the optimal values which are easy to use and inexpensive. We approximate $\alpha$ and $\beta$ by constants. The key point now is how to choose $\alpha$ and $\beta$ so that the obtained new WR algorithm converges fast and much faster than the cWR algorithm that is known to be very slow for transmission line problems. Mathematically, we want $\alpha$ and $\beta$ that make $\left|\rho_{\text {opt }}\right| \ll 1$. To do so, we have solved the optimization problem

$$
\min _{\alpha, \beta}\left(\max _{\mathcal{R}(s) \geq 0}\left|\rho_{\text {opt }}(s, a, b, \tilde{b}, c, \alpha, \beta)\right|\right) .
$$

From the optimal choice (II.4) one can see that $\beta=-\frac{1}{\alpha}$, and since the circuit considered here behaves identically in both sides of the partition, we simplify the optimization process by taking $\alpha=-\frac{1}{\beta}$. Further, using the fact that $\rho_{\text {opt }}$ is analytic in the right half of the complex plane provided $\alpha<0$ and $\beta>0$, by the maximum principle we have that the maximum of $\left|\rho_{\text {opt }}\right|$ is on the boundary, i.e., at $\sigma=0$. Moreover, the modulus of the convergence factor $\rho_{\text {opt }}$ is symmetric about the real axis, and hence, it is sufficient to consider non-negative frequencies. Therefore, our min-max problem can be reduced to

$$
\min _{\alpha<0}\left(\max _{0 \leq \omega<\infty}\left|\rho_{\text {opt }}(i \omega, a, b, \tilde{b}, c, \alpha)\right|\right) .
$$

Our extensive numerical experiments have shown that the solution of the min-max problem (II.5) occurs when $\left|\rho_{\text {opt }}\right|$ at $\omega=0$ and that at $\omega=\bar{\omega}$ are balanced, where $\bar{\omega}$ is the interior maximum of $\left|\rho_{\text {opt }}\right|$. Therefore, $\alpha^{*}$ is characterized by the equation

$$
\left|\rho_{\text {opt }}\left(0, a, b, \tilde{b}, c, \alpha^{*}\right)\right|=\left|\rho_{\text {opt }}\left(\bar{\omega}, a, b, \tilde{b}, c, \alpha^{*}\right)\right|,
$$

independently of the transient analysis time $T$. It should be noted that for the RLC circuit case considered in [17], there was a dependence on $T$ when solving the min-max problem analyzed there. In Fig. 2, we give an example for the modulus of the classical convergence factor and the optimized convergence factor from the numerical section, using the

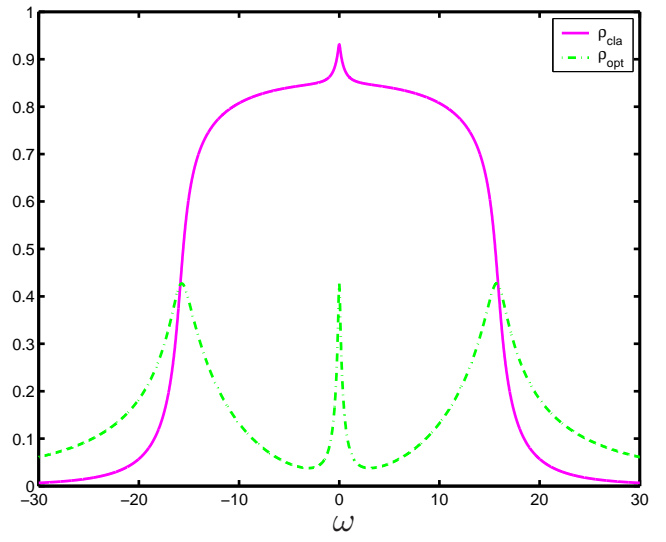

Fig. 2. Optimized convergence factor versus classical one.

optimized value $\alpha^{*}=-0.5426$. The equioscillation in $\rho_{\text {opt }}$ at $\omega=0$ and $\omega=\bar{\omega}$ is clear from Fig. 2. Further, one can see that $\left|\rho_{\text {cla }}\right|$ is not uniform and has a maximum of about 0.9317. Whereas $\left|\rho_{\text {opt }}\right|$ shows a remarkable improvement in magnitude and uniformity and has a maximum of only about 0.4272 .

\section{NUMERICAL EXPERIMENTS}

We consider in this section two examples. The first one is a small RLCG TL model which is $1 \mathrm{~cm}$ long with 4 sections, in order to show that the proposed oWR algorithm works well for small TLs. The second example is a large RLCG TL model, where we choose a $10 \mathrm{~cm}$ long TL circuit with 200 sections. We use the source term $I_{s}(t)=10 t \mathrm{~mA}$, for $0<t<0.1 \mathrm{~ns}$, and $I_{s}=1 \mathrm{~mA}$ for $t \geq 0.1 \mathrm{~ns}$, and the analysis time interval is $t \in[0, T]$, with transient analysis time $T=20 \mathrm{~ns}$. For the circuit elements per unit length, we use $R=0.25 \Omega / \mathrm{cm}, L=4 \mathrm{nH} / \mathrm{cm}, C=1.6 \mathrm{pF} / \mathrm{cm}$, and $G=2 \mathrm{mho} / \mathrm{cm}$. The load and termination resistances are chosen to be $50 \Omega$. To integrate the equations in time, we use the backward Euler method with 500 time steps, i.e., $\Delta t=\frac{T}{500}$. We use zero initial conditions and random initial waveforms. The optimized parameters $\alpha^{*}=-0.5426$ and $\beta^{*}=-\frac{1}{\alpha^{*}}$ are used in the oWR algorithm. To illustrate the difference in convergence between the cWR and oWR algorithms analyzed in this paper, we show in Fig. 3 the error as a function of iterations measured in infinity norm between the converged solution computed using the entire circuit, i.e., by a conventional Spice type approach, and the iterates. We show the error for the small circuit example on the left while 

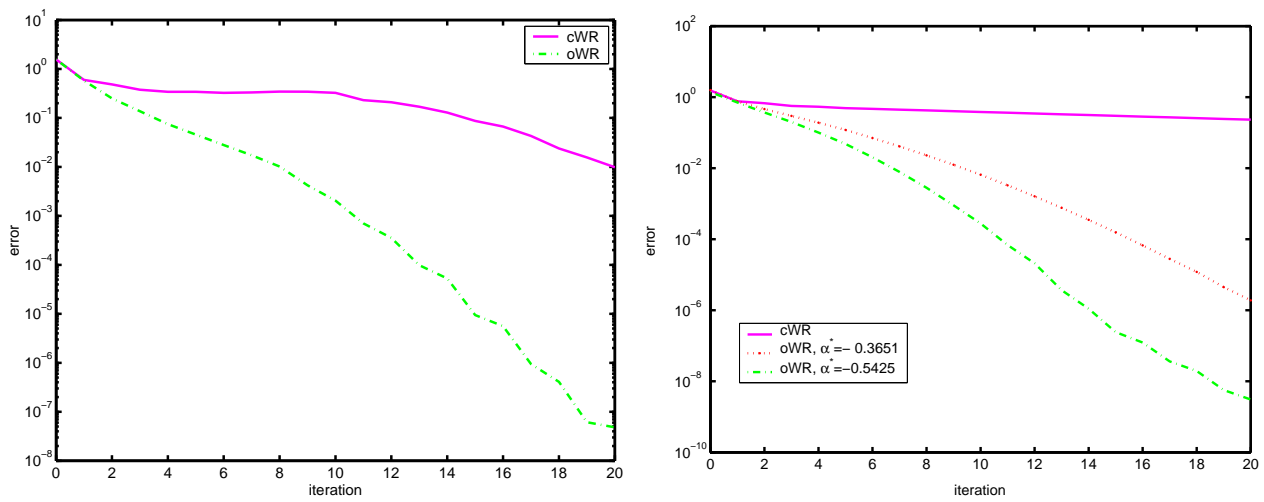

Fig. 3. Convergence behavior of the cWR algorithm compared to the oWR algorithms.Left: small circuit. Right: large circuit
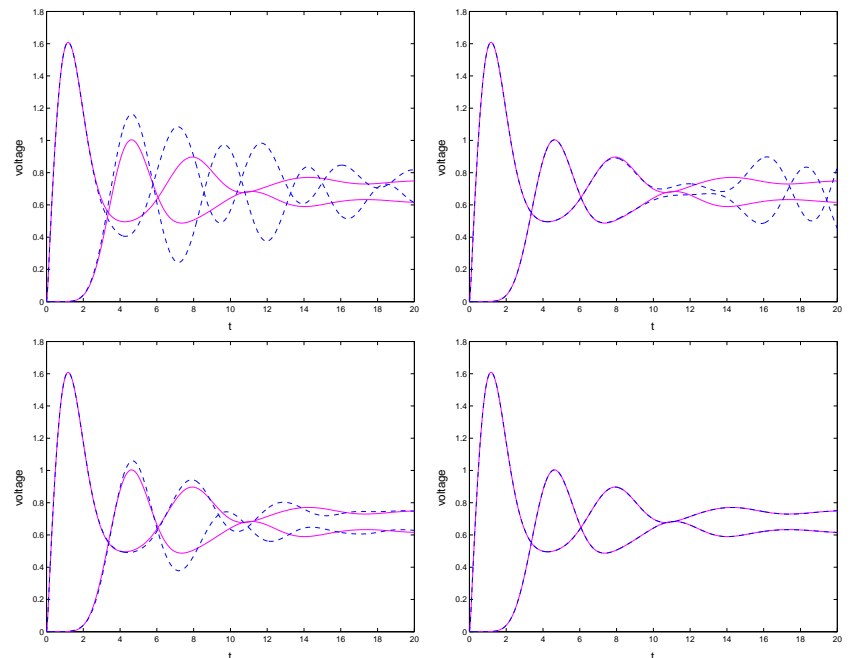

Fig. 4. The first and last voltage values, the exact values in solid line, and in dashed line on the top, the iterates 3 and 10 for cWR algorithm, and on the bottom for oWR algorithm.

we give the results for the large circuit on the right. We can observe better convergence for the new oWR algorithms than the cWR algorithm which has difficulties to converge in both examples. Note that we include on the right of Fig. 3 the error of the oWR using the optimized parameters $\alpha^{*}=-0.3651$ and $\beta^{*}=-\frac{1}{\alpha^{*}}$ from [17] for comparison purposes. It is clear that the new oWR algorithm presented here is better than the one introduced in [17] when the TL model is the RLCG type model. We finally show in Fig. 4 the first and last voltage values over time. The solid line represents the converged solution in all graphs. On the top, we show the results from the cWR algorithm with dashed lines, while the bottom represents the oWR algorithm. We observe that the convergence for the cWR algorithm is slow, and much faster convergence is obtained by the new oWR algorithm, where an accurate result is obtained in about 10 iterations without subdivision of the time into time windows.

\section{CONCLUSIONS}

In this paper, we included the shunt loss $G$ in the transmission line model for the oWR approach for the difficult longitudinal partitioning problem. We presented a practical oWR algorithm that is inexpensive and easy to use. Also, it greatly improves the convergence for a single RLCG line type circuit. It could also be directly applied to multiple lines in conjunction with the transverse partitioning technique. The results show the better performance of the new oWR algorithm for an RLCG model over the cWR. To the best of our knowledge, this is the first time that such algorithms have been proposed and analyzed for TL models with shunt resistences $G$.

\section{REFERENCES}

[1] E. Lelarasmee, A. Ruehli, and A. L. Sangiovanni-Vincentelli. Waveform relaxation decoupling method. IBM Technical Disclosure Bulletin, 24(7B):3720-3721, December 1981.

[2] E. Lelarasmee, A. E. Ruehli, and A. L. Sangiovanni-Vincentelli. The waveform relaxation method for time-domain anaylsis of large-scale integrated circuits. IEEE Trans. on CAD of Integrated Circuits and Systems, CAD-1(3):131-145, July 1982.

[3] J. White, A. Sangiovanni-Vincentelli, F. Odeh, and A. Ruehli. Waveform relaxation: theory and practise. Trans. Soc. Computer Simulation, 2:95$133,1985$.

[4] J. White and A. Sangiovanni-Vincentelli. Relaxation techniques for the simulation of VLSI circuits. Kluwer Academic Publishers, Boston, 1987.

[5] Y.-L. Jiang, R. M. M. Chen, and O. Wing. Convergence analysis of waveform relaxation for nonlinear differential-algebraic equations of index one. IEEE Trans. Circuits Systems I Fund. Theory Appl., 47(11):1639-1645, 2000.

[6] W. T. Beyene. Application of multilinear and waveform relaxation methods for efficient simulation of interconnect-dominated nonlinear networks. IEEE Transactions on Advanced Packaging, 31(3):637-648, 2008.

[7] R. Jeltsch and B. Pohl. Waveform relaxation with overlapping splittings. SIAM J. Sci. Comput., 16(1):40-49, 1995.

[8] M. Bjorhus and A. M. Stuart. Waveform relaxation as a dynamical system. Mathematics of Computation, 66(219):1101-1117, July 1997.

[9] M. J. Gander and A. M. Stuart. Space-time continuous analysis of waveform relaxation for the heat equation. SIAM Journal on Scientific Computing, 19(6):2014-2031, 1998.

[10] M. J. Gander, L. Halpern, and F. Nataf. Optimal Schwarz waveform relaxation for the one dimensional wave equation. SIAM Journal on Numerical Analysis, 41(5):1643-1681, 2003.

[11] M. J. Gander and L. Halpern. Absorbing boundary conditions for the wave equation and parallel computing. Math. of Comp., 74(249):153176, 2004. 
[12] M. J. Gander and L. Halpern. Optimized Schwarz waveform relaxation methods for advection reaction diffusion problems. SIAM J. Numer. Anal., 45(2):666-697, 2007.

[13] D. Bennequin, M. J. Gander, and L. Halpern. A homographic best approximation problem with application to optimized Schwarz waveform relaxation. Math. Comp., 78:185-223, 2009.

[14] V. B. Dmitriev-Zdorov. Generalized coupling as a way to improve the convergence in relaxation-based solvers. In Design Automation Conference, 1996, with EURO-VHDL '96 and Exhibition, Proceedings EURO-DAC '96, European, 1996. Held in Geneva, Switzerland, Sept. 1996.

[15] M. J. Gander and A. E. Ruehli. Optimized waveform relaxation methods for RC type circuits. IEEE Transaction on Circuits and Systems, 51(4):755-768, April 2004.

[16] M. J. Gander and A. E. Ruehli. Solution of large transmission line type circuits using a new optimized waveform relaxation partitioning. IEEE International Symposium on Electromagnetic Compatibility, 2:636-641, August 2003.

[17] M. J. Gander, M. Al-Khaleel, and A. Ruehli. Optimized waveform relaxation methods for longitudinal partitioning of transmission lines. IEEE Transactions on Circuits and Systems, I, 56(8):1732-1743, August 2009.

[18] M. J. Gander and A. E. Ruehli. Optimized waveform relaxation solution of electromagnetic and circuit problems. In Digest of Electr. Perf. Electronic Packaging, volume 19, pages 65-68, Austin, TX, October 2010
[19] Y. Courvoisier and M. J. Gander. Time domain Maxwell equations and Schwarz waveform relaxation methods. In R. E. Bank, M. J. Holst, O. B. Widlund, and J. Xu, editors, Domain Decomposition Methods in Science and Engineering XX. Springer LNCSE, 2012.

[20] A. Alonso-Rodriguez and L. Gerardo-Giorda. New nonoverlapping domain decomposition methods for the harmonic Maxwell system. SIAM J. Sci. Comput., 28(1):102-122, 2006.

[21] V. Dolean, M. J. Gander, and L. Gerardo-Giorda. Optimized Schwarz methods for Maxwell equations. SIAM J. Scient. Comp., 31(3):21932213, 2009.

[22] V. Rawat and J.-F. Lee. Nonoverlapping domain decomposition with second order transmission condition for the time-harmonic Maxwell's equations. SIAM J. Sci. Comput., 32(6):3584-3603, 2010.

[23] Z. Peng and J.-F. Lee. Non-conformal domain decomposition method with second-order transmission conditions for time-harmonic electromagnetics. J. Comput. Phys., 229(16):5615-5629, 2010.

[24] M. El Bouajaji, V. Dolean, M. J. Gander, and S. Lanteri. Optimized Schwarz methods for the time-harmonic Maxwell equations with dampimg. SIAM J. Scient. Comp., 34(4):2048-2071, 2012.

[25] N. M. Nakhla, A. E. Ruehli, M. S. Nakhla, R. Achar, and C. Chen. Waveform relaxation techniques for simulation of coupled interconnects with frequency-dependent parameters. IEEE Transactions on Advanced Packaging, 30(2):257-269, May 2007.

[26] X. Ji, L. Ge, and Z. Wang. Analysis of on-chip distributed interconnects based on Pade expansion. J. of Control theory and Appl., 7(1):92-96, 2009. 